\title{
Induction of lymphocytes activated marker CD69 following exposure to chitosan and alginate biopolymers
}

\author{
Olga Borges ${ }^{a, *}$, Gerrit Borchard ${ }^{b}$, Adriano de Sousa ${ }^{a}$, \\ Hans E. Junginger ${ }^{\mathrm{c}}$, Anabela Cordeiro-da-Silva ${ }^{\mathrm{d}}$ \\ a Center for Pharmaceutical Studies and Laboratory of Pharmaceutical Technology, Faculty of Pharmacy, \\ University of Coimbra, Rua do Norte, 3000-295 Coimbra, Portugal \\ b School of Pharmacy Geneva/Lausanne, University of Geneva, 1211 Geneva 4, Switzerland \\ c Faculty of Pharmaceutical Sciences, Naresuan University, Phitsanulok 65000, Thailand \\ ${ }^{\mathrm{d}}$ Institute for Molecular and Cell Biology and Laboratory of Biochemistry, Faculty of Pharmacy, University of Porto, $4050-047$ Porto, Portugal
}

Received 16 November 2006; received in revised form 4 January 2007; accepted 13 January 2007

Available online 20 January 2007

\begin{abstract}
CD69 is a very early cell activation antigen expressed on the surface of activated immune cells. It can appear within $1-2 \mathrm{~h}$ of activation and exhibits maximal expression levels between 18 and $24 \mathrm{~h}$ after stimulation. In this work, the expression profile of CD69 in mice splenocytes was evaluated following exposure to the biopolymers, chitosan or alginate and the immunostimulatory factors, CpG ODN 1826 or concanavalin A. We have shown that both polymers are able to upregulate expression of CD69 on B cells and CD4+ T-lymphocytes, with alginate as the least potent stimulus. Moreover, the expression of the CD69 molecule on CD8+ T-lymphocytes was observed only in splenocytes cultured with chitosan. However, activation of lymphocytes did not result in cell proliferation. On the other hand, CpG ODN proved to be a potent B cell stimulator, as evidenced by the upregulation of CD69, but had less effect on T-cells. These results, together with previous discoveries reported in scientific literature, may contribute to the clarification of the adjuvant effect, which has been attributed to chitosan and alginate formulations or to the biopolymers itself.
\end{abstract}

(C) 2007 Elsevier B.V. All rights reserved.

Keywords: CD69 expression; Chitosan; Alginate; CpG ODN; Lymphocyte activation

\section{Introduction}

The combination of biomaterials with proteins, DNA, vaccines, and cells has been a matter of study in several groups. Two well-known examples are the development of polymeric carriers of vaccines and tissue engineered constructs in which cells are delivered with a polymer component for the renovation or replacement of damaged tissue or organ function. In these two examples, different potential immune responses, due to the possible adjuvant effect of the biomaterial would be required. Clearly, from a tissue engineering point of view, immune responses are to be minimized or altogether avoided while an adjuvant effect of the polymeric vaccine delivery system would be desirable and even necessary, particularly for weak

\footnotetext{
* Corresponding author. Tel.: +351 239859927; fax: +351 239827126.

E-mail address: olga@ci.uc.pt (O. Borges).
}

antigens (Babensee and Paranjpe, 2005). Among the natural biomaterials, chitosan and alginate have been used for both of the previously stated purposes. In particularly the use of alginate (Kim et al., 2002; Suckow et al., 2002; Tafaghodi et al., 2006) and chitosan (van der Lubben et al., 2003; Xu et al., 2004; Baudner et al., 2005; Read et al., 2005) particles as an antigen delivery system were used with success in a number of vaccination studies. The mechanism, by which particulates showing adjuvant effect is not completely understood. In general, are thought that particulate delivery systems promote trapping and retention of antigens in local lymph nodes and protecting them from degradation, which seems to be important in triggering protective T cell responses (O'Hagan, 2006). However in a recent study (Read et al., 2005), a soluble derivative of the chitosan was administered in solution with influenza vaccine. An adjuvant effect of the chitosan was observed; therefore a different mechanism in this case may have to be considered and investigated. 
We also have been particularly interested in the use of chitosan and alginate for the design of vaccine carriers. In a recent publication (Borges et al., 2005), we described the development of a new vaccine delivery system of alginate coated chitosan nanoparticles, specially designed to deliver vaccines into mucosal surfaces. This delivery system is now being used in mucosal vaccination studies with the recombinant hepatitis B surface antigen associated with the adjuvant, the CpG ODN 1826. By this reason, the study of the effect of the polymers, used in our group for the preparation of the nanoparticles, on the cells from the immune system are of extreme importance.

Both polymers are naturally occurring polysaccharides. Chitosan, a copolymer of D-glucosamine and $N$-acetyl-Dglucosamine is a derivative of chitin, one of the most abundant polysaccharides in nature. Alginate is composed of alternating blocks of 1-4 linked $\alpha$-L-guluronic (G) and $\beta$-D-mannuronic (M) acid residues. The sources of these polymers as well as their physical and chemical properties have been extensively reviewed in several publications (Felt et al., 1998; van der Lubben et al., 2001; George and Abraham, 2006).

The immunostimulatory properties, found in certain alginates, in same cases have been associated with the presence of impurities, like endotoxins or mitogenic contaminants in alginate raw materials (Klock et al., 1997; Jork et al., 2000; Orive et al., 2005). In a recent work, the production of the proinflammatory cytokine TNF- $\alpha$ was approximately 100 times higher in the case of non-biomedical grade alginate in comparison to the purified one (Orive et al., 2005). According to the same authors, this feature has implications on the utilization of impure alginates, for instance as carriers for cell immobilization. In fact, TNF- $\alpha$ is considered to be a potent cytokine that is known to activate leukocytes, stimulate fibroblast proliferation, promote migration of inflammatory cells into the intercellular matrix, and trigger local secretion of other proinflammatory cytokines. In addition to the considerations above, a vaccine nanocarrier should also be made with purified alginates given that stimulation of TNF- $\alpha$ would lead to an overgrowth of collagen-secreting fibroblasts and activated macrophages secreting growth regulatory cytokines over the nanocarrier and may have as a consequence, an alteration of the vaccine release from the nanocarrier (Orive et al., 2005). On other hand, a highly purified alginate, with low polyphenol and protein content, prepared from Laminaria pallida, was evaluated in vitro using mice lymphocytes and no evidence of significant mitogenic activity was observed (Jork et al., 2000). However there are also some studies that showed that the mannuronic acid rich alginates, have itself immunostimulating properties, stimulating monocytes to cytokine production (Otterlei et al., 1993) in where the TLR2 and TLR4 seemed to be involved (Flo et al., 2002). In a more recent study (Babensee and Paranjpe, 2005), the effect of biomaterials on dendritic cell maturation was evaluated and it was demonstrated that the expression levels of CD86, CD40 and HLA-DQ (MHC class II) molecules, indicative of dendritic cell maturation, were decreased in the presence of alginate. On the contrary, the presence of chitosan in the same in vitro study caused a high expression level of the same co-stimulatory molecules.
The expression of CD69 was never investigated in lymphocytes cultured with chitosan; however the activation of lymphocytes following the administration of chitosan has been reported in some recent publications. For instance, it was shown that rats fed orally with a purified low molecular weight chitosan triggered the release of IL-10 as well as the expression of IL- 4 and TGF- $\beta$ mRNA at the gut mucosa local microenvironment and stimulated $\mathrm{CD} 3+\mathrm{T}$-lymphocytes in the spleen (Porporatto et al., 2005). The presence of chitosan also caused the enhancement of the natural killer (NK) cell activity in intestinal intraepithelial lymphocytes and splenic lymphocytes (Maeda and Kimura, 2004). Furthermore, it has been demonstrated that chitosan (Bianco et al., 2000; Mori et al., 2005), and chitosan microparticles (Nishimura et al., 1987; Shibata et al., 1997) are able to up-regulate, to some extent, a number of macrophage functions. For instance, a low molecular weight water soluble chitosan induced the activation of macrophages through the production of cytokines such as IFN- $\gamma$, IL-12, and IL-18 from the intestinal intraepithelial lymphocytes (Maeda and Kimura, 2004). Another example found in Scientific literature based on in vitro studies, shown that chitosan nanoparticles $(1-10 \mu \mathrm{m})$ that can be phagocytized, stimulated alveolar macrophages, but at a significantly lower level than that elicited by oxidative responses observed with phagocytized chitin (Shibata et al., 1997). In a more recent study (Mori et al., 2005), using peritoneal macrophages, different results among the biomaterials chitosan, low molecular weight chitosan (LMW), and chitin were obtained. Therefore, chitosan treatment induced activation markers, such as the major histocompatibility complex (MHC) class I, class II, Fc receptors, transferrin receptor, mannose receptor, Fas, and macrophage inflammatory protein (MIP)-2, whereas chitin and LMW chitosan induced only the expression of MHC class I and II molecules. Thus, small variations in molecular weight or degree of deacetylation on chitosan molecule might lead to different degrees of activation in cells from the immune system (Ueno et al., 2001; Matsunaga et al., 2006).

The adjuvant effects of $\mathrm{CpG}$ ODN have been studied intensively in the last few years. Briefly, CpG ODN directly stimulates immune cell types that express Toll-like receptor 9 (TLR9), with human B cells and plasmocytoid dendritic cells ( $\mathrm{pDCs}$ ) being the dominant cell types expressing TLR9 (Klinman, 2004). Activation of these cells promotes the production of Thelper 1 (Th1) and proinflammatory cytokines and the maturation/activation of professional antigen-presenting cells (APC) (Klinman, 2004). These actions enable $\mathrm{CpG}$ ODNs to act as immune adjuvants which can accelerate and boost antigen-specific immune responses. The maintenance of close physical contact between the $\mathrm{CpG}$ and the antigen has been reported to be the ideal situation for achieving optimal results (Davis et al., 1998). This can be realized by encapsulating the $\mathrm{CpG}$ and the antigen together in the same delivery system. Moreover, if the polymers used to construct the delivery system, for instance chitosan and alginate, have themselves immune stimulating properties, then the formulation, may have optimized adjuvant properties.

Therefore, the main goal of this work was to evaluate the capacity of each component of our coated nanoparticles, and 
compare them with the adjuvant, CpG ODN 1826, in order to determine which method resulted in the optimal activation of Band T-lymphocytes. With this purpose, the CD69 receptor, which is considered a very early cell activation antigen, expressed on the surface of activated immune cells, was examined for the first time on splenocytes cultured with the raw materials chitosan or alginate, which are used in the preparation of the coated nanoparticles. This cell surface antigen can appear within 1-2 h of activation and exhibits maximal expression levels between 18 and $24 \mathrm{~h}$ after stimulation, making it a useful tool, albeit one that has not been fully investigated, for early evaluation of the effect of biomaterials in the stimulation of immune cells.

\section{Materials and methods}

\subsection{Materials}

\subsubsection{Animals}

In this study, 6-7-week-old male BALB/c mice were used (Harlan Iberica, Barcelona, Spain). Animals were housed and cared for at the animal resource facilities of the Faculty of Pharmacy of the University of Porto, in accordance with institutional guidelines.

\subsubsection{Polymers}

Fine grade pure chitosan was purchased from Primex BioChemicals AS (Avaldsnes, Norway). According to the provider's specifications, the degree of deacetylation is $95 \%$ (titration method) and the viscosity is $8 \mathrm{cP}$ (measured in $1 \%$ solutions in $1 \%$ acetic acid) that corresponds to a low molecular weight chitosan. (According to the provider's specifications a chitosan with a molecular of $40 \mathrm{kDa}$ and another with $60 \mathrm{kDa}$ have a viscosity of 25 and $35 \mathrm{cP}$, respectively.)

A low molecular weight sodium alginate (MANUCOL LB ${ }^{\circledR}$ ) was kindly donated by ISP Technologies Inc. (Surrey, UK). According to the provider's specifications, the typical values for the percentage of mannuronic and guluronic acid for Manucol LB is 61 and 39\%, respectively, and an estimated molecular weight of $18 \mathrm{kDa}$.

Both polymers meet the requisites described in the European Pharmacopoeia for use in pharmaceutical formulations. In this work a $22 \mu \mathrm{m}$ filtered solutions of the polymers were used.

\subsubsection{Reagents}

Class B, CpG ODN 1826 (5'-TCC ATG ACG TTC CTG ACG TT-3') was purchased from Coley Pharmaceutical Group (Ottawa, Canada), concanavalin A (Con A) was purchased from Sigma Chemicals (St. Louis, USA), certified fetal bovine serum (FBS) and L-glutamine $(200 \mathrm{mM})$ were purchased from Gibco (Invitrogen Co., Paisley, Scotland, UK), 1 M HEPES buffer $(0.85 \% \mathrm{NaCl})$, RPMI 1640 medium without L-glutamine and Pen-Strep (10,000 U penicillin/ml; 10,000 $\mu \mathrm{g}$ streptomycin $/ \mathrm{ml}$ ) were purchased from Biowhitaker (Cambrex Bio Science, Verviers, Belgium). [Methyl- ${ }^{3} \mathrm{H}$ ] thymidine $(1.0 \mathrm{mCi} / \mathrm{ml})$ was obtained from Amersham Biosciences (UK), R-Phycoerythrin (PE)-conjugated hamster anti-mouse CD69, fluorescein isothiocyanate (FITC)-conjugated rat anti-mouse
CD4 and FITC-conjugated rat anti-mouse CD8 were obtained from BD Biosciences (Madrid, Spain). The FITC-conjugated goat anti-mouse IgM (anti- $\mu$ ) was purchased from PharMingen (San Diego, CA, USA). All others reagents used were of analytical grade. All solutions were prepared in Millipore water.

\subsection{Methods}

\subsubsection{Preparation of spleen cell suspensions}

Three mice were euthanized by cervical dislocation and their spleens were aseptically removed. Individual spleen cell suspensions were prepared in a Petri dish using curved needles and washed twice with RPMI 1640. The final suspension was adjusted to a final concentration of $1 \times 10^{7}$ cells $/ \mathrm{ml}$ in complete RPMI 1640 medium (supplemented with $10 \%$ (v/v) fetal bovine serum, $1 \%$ (v/v) glutamine, $1 \%$ (v/v) Pen-Strep and 2\% (v/v) $1 \mathrm{M}$ HEPES buffer).

\subsubsection{Polymer solutions}

The $0.25 \%(\mathrm{w} / \mathrm{v}), 0.125 \%(\mathrm{v} / \mathrm{v})$, and $0.01 \%(\mathrm{v} / \mathrm{v})$ chitosan solutions were prepared in complete RPMI medium acidified with acetic acid. Sodium alginate was first dissolved in HEPES buffer $(0.5 \%, \mathrm{w} / \mathrm{v})$ and then diluted in complete RPMI $(0.25 \%$, $\mathrm{v} / \mathrm{v}), 0.125 \%(\mathrm{v} / \mathrm{v})$, and $0.01 \%(\mathrm{v} / \mathrm{v}))$. Immediately following, the polymer solutions were aseptically filtered and incubated with the cells. The solvents used for the preparation of the polymer solutions (RPMI acidified with acetic acid and HEPES) were also used as controls for the experiment.

\subsubsection{Cell culture in the presence of the polymers and mitogens}

Using sterile 96-well flat-bottomed tissue culture plates, $25 \mu \mathrm{l}$ of splenocyte suspension $\left(1 \times 10^{7}\right.$ cells $\left./ \mathrm{ml}\right)$ from three individual mice were plated in triplicate along with $25 \mu \mathrm{l}$ of complete RPMI solution with the mitogen [Con A $(50 \mu \mathrm{g} / \mathrm{ml})$ or CpG ODN $(50 \mu \mathrm{g} / \mathrm{ml})$ or without mitogen (control)] and $25 \mu \mathrm{l}$ of a complete RPMI solution of the polymer [chitosan; or sodium alginate; or without polymer (control)]. Finally the volume of the well was diluted to $200 \mu \mathrm{l}$ with complete RPMI and incubated at $37^{\circ} \mathrm{C}$ with $95 \%$ relative humidity and in the presence of $5 \% \mathrm{CO}_{2}$.

\subsubsection{Immunofluorescence labelling}

After $20 \mathrm{~h}$ of incubation, cells were washed and resuspended in cold phosphate buffered saline (PBS) supplemented with $2 \% \mathrm{FBS}$, resulting in a concentration of $0.5 \times 10^{6}$ cells/well. The cells were then incubated in the dark for $30 \mathrm{~min}$ at $4{ }^{\circ} \mathrm{C}$ with saturating concentrations of PE-conjugated hamster antimouse CD69 and FITC-conjugated rat anti-mouse CD4 or FITC-conjugated rat anti-mouse CD8 or FITC-conjugated goat anti-mouse IGM (anti- $\mu$ ). After incubation, cells were washed three times with PBS-FBS and then resuspended with $500 \mu \mathrm{l}$ of PBS-FBS. To exclude dead cells, $2.5 \mu$ l propidium iodide $(50 \mu \mathrm{l} / \mathrm{ml})$ was added just before data acquisition. At least 10,000 events were analysed by flow cytometric acquisition, performed 
in a fluorescent activated cell sorter (FACS Calibur) (BD Biosciences, Madrid, Spain). Data were analysed by CellQuest software (BD Biosciences, Madrid, Spain). Viable lymphocytes were selected on the basis of FSC/SSC values.

The concentration of the polymers studied was kept at $0.156 \mathrm{mg} / \mathrm{ml}$. Data were presented as the mean \pm S.E.M. for three experiments and statistical significance was assessed using repeated measured one-way ANOVA followed by Dunnett's post-test using the GraphPad Prism 4 software. Differences were considered significant when $p<0.05$.

\subsubsection{Lymphocytes proliferation assay}

Splenocytes were obtained and cultured in a flat-bottomed 96-well plate with and without the mitogens and polymers as described above. The cells were cultured for $96 \mathrm{~h}$ at $37^{\circ} \mathrm{C}$ and on the last $8 \mathrm{~h}$ of incubation, each well was pulsed with $1 \mu \mathrm{Ci}$ of [methyl $-{ }^{3} \mathrm{H}$ ] thymidine. 96 well plates with the cells were frozen for further analysis. The cells were later thawed and harvested onto a Fiberglass filter (filter mats, molecular devices, Skatron, Lier, Norway) using a semiautomatic cell harvester (Scatron Instruments, USA) and thymidine incorporation was counted by standard liquid scintillation techniques with a Beckman LS 6500 scintillation counter (Beckman Coulter Inc., Fullerton, USA). Thymidine incorporation was expressed as counts per minute (cpm).

The concentration in the well of the polymers studied were: $\operatorname{conc} 1=0.312 \mathrm{mg} / \mathrm{ml}$ and $\operatorname{conc} 2=0.0125 \mathrm{mg} / \mathrm{ml}$. Data were presented as a mean \pm S.E.M. for the three experiments and statistical significance was assessed using a nonparametric ANOVA with Kruskal-Wallis analysis using the GraphPad
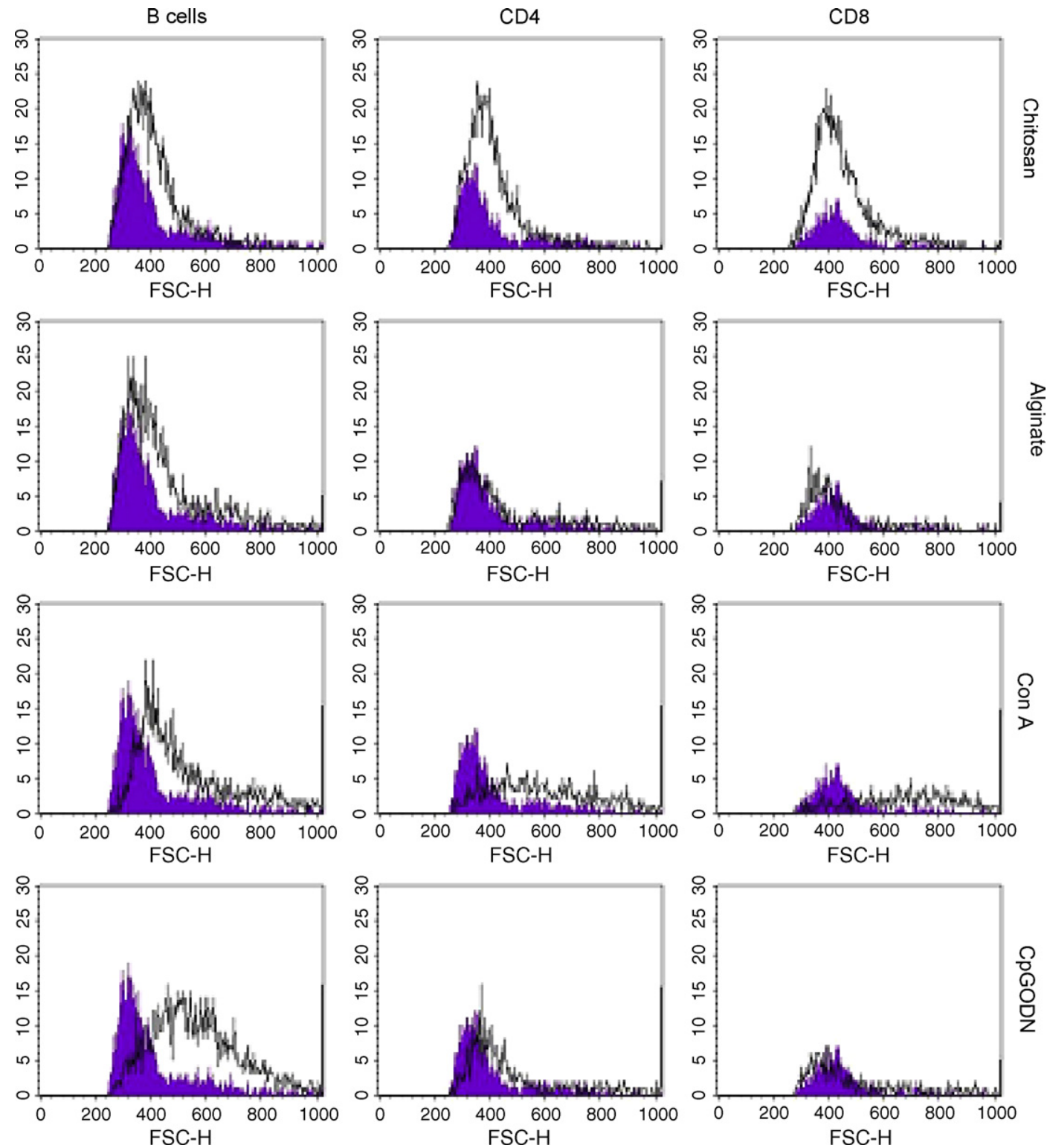

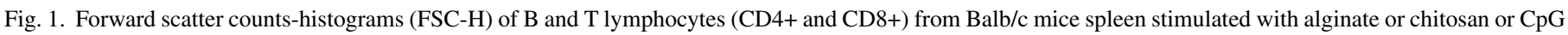

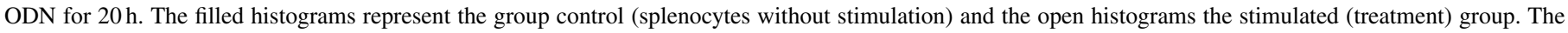
results are representative of two separate experiments using three animals per experiment and each treatment. 
Prism 4 software. Differences were considered significant when $p<0.05$.

\section{Results}

\subsection{Effect of alginate or chitosan polymers or the immunostimulant $\mathrm{Cp} G \mathrm{ODN}$ on the size and granularity of spleen mouse lymphocytes}

The spleen is a lymphoid organ combining the innate and adaptive immune system in a unique and organized way (Mebius and Kraal, 2005). The principal reason for the choice of spleen cells in this study was related to the fact that they are obtained and cultured easily and that they are a very sensitive representative of different lymphocytes. According to FACS analysis performed in our own laboratory, a primary culture of mixed spleen cells normally contain more than $50 \%$ of B lymphocytes, more than $30 \%$ of $\mathrm{T}$ lymphocytes $(20 \% \mathrm{CD} 4+; 12 \% \mathrm{CD} 8+)$ and of the remaining cells, less than $10 \%$ were erythrocytes, fibroblasts, macrophages, dendritic cells, and granulocytes.

Lymphocytic morphological alterations, as a consequence of the engorgement of the rough endoplasmic reticulum, which normally accompany cell activation, can be observed by flow cytometry analysis, combining forward light scatter (FSC) and side light scatter (SSC). In general, activated cells exhibited a dramatic increase in both forward and side scatter.

As a positive control, cells from the mouse spleen were cultured with concanavalin A (Con A), a classical lymphocyte mitogen. As was expected and shown in Fig. 1, an increase of the forward scatter counts (FSC) of the stimulated lymphocytes was observed, suggesting augmentation of cell size. The size change was accompanied by an increase in cellular granularity
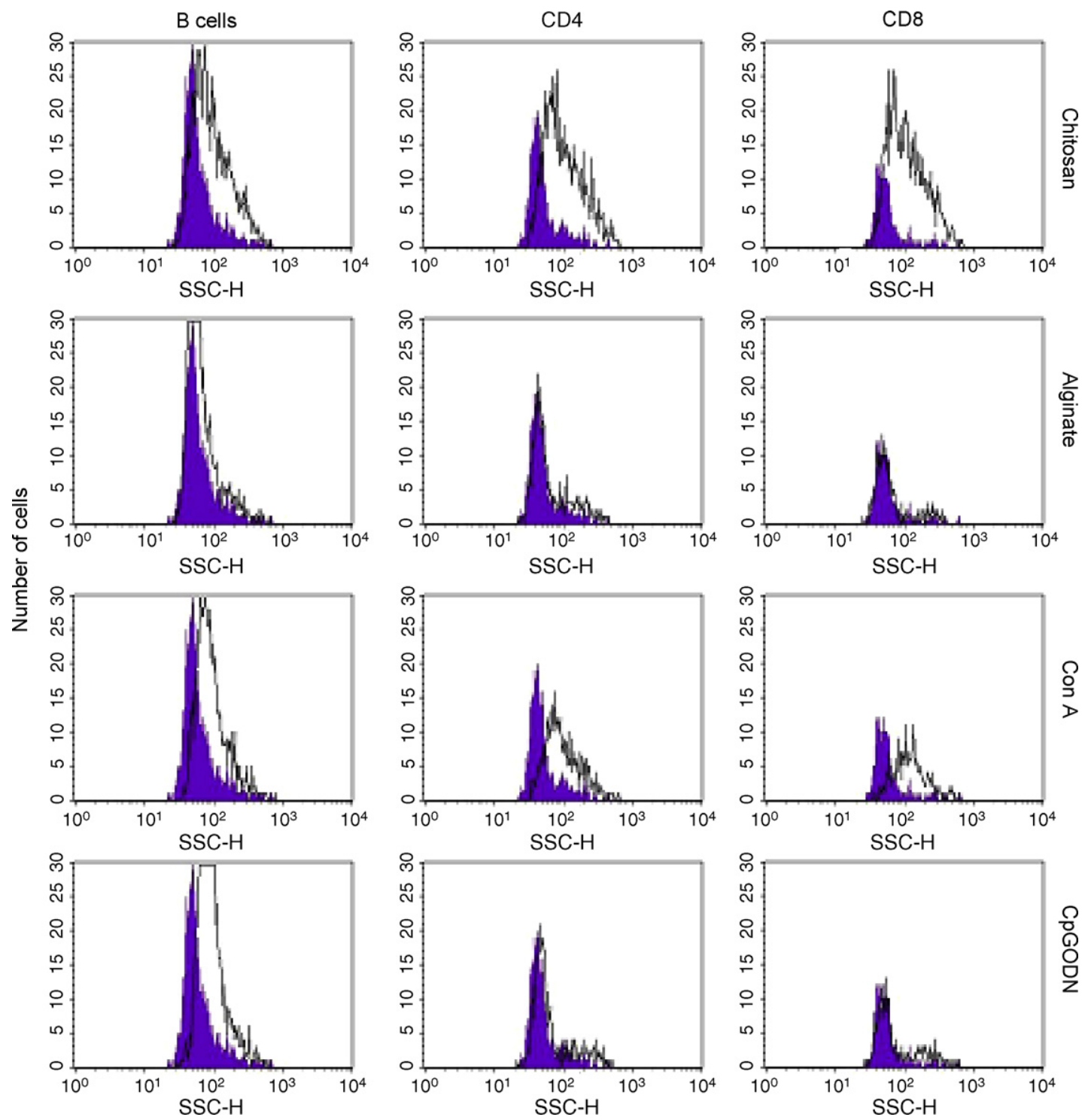

Fig. 2. Side light scatter counts-histograms (SSC-H) of B and T lymphocytes (CD4+ and CD8+) from Balb/c mice spleen stimulated with alginate or chitosan or CpG ODN for $20 \mathrm{~h}$. The filled histograms represent the group control (splenocytes without stimulation) and the open histograms the stimulated (treatment) group. The results are representative of two separate experiments using three animals per experiment and each treatment. 
(SSC) in all lymphocyte subtypes (CD4+, CD8+, and B cells) after $20 \mathrm{~h}$ culture (Fig. 2). In contrast, the presence of CpG ODN in the splenocytes culture, subjected to the same conditions did not induce a size (Fig. 1) and granularity (Fig. 2) augmentation in CD4+ and CD8+ T-cells, however, a remarkable increase of these two parameters was observed in B cells. These results support the hypothesis that $\mathrm{CpG}$ ODN is predominately a $\mathrm{B}$ lymphocyte activator.

In the same way, to assess the differential effect of the alginate and chitosan biopolymer contact on spleen cell morphology, the cells were cultured with the polymers for $20 \mathrm{~h}$. Preliminary results (Borges et al., 2006) have shown cell viability near $100 \%$ with splenocytes cultured with $0.21 \mathrm{mg} / \mathrm{ml}$ of the polymers for $15 \mathrm{~h}$. In order to obtain non-toxic concentrations of polymers, we decided to decrease the concentration of polymers in the cell culture to $0.156 \mathrm{mg} / \mathrm{ml}$ because we increased the incubation time to $20 \mathrm{~h}$.

As shown in Figs. 1 and 2, both polymers caused an increase in FSC and SSC of B cells. Chitosan was the polymer that showed a stronger effect, particularly on cell granularity (SSC). Moreover, CD4+ and CD8+ T-lymphocytes treated with chitosan also showed a strong modification of their morphology (SSC and FSC). T-lymphocytes treated with alginate did not show morphological differences (SSC and FSC) when compared to the appropriate control (filled overlap histogram in Figs. 1 and 2).

\subsection{Evaluation of the expression of CD69 by $T$ and $B$ mouse spleen lymphocytes}

As a positive control, we used Con A to induce the expression of CD69 in B and T lymphocytes and the results are shown in Figs. 3-5. The CD69 expression results in spleen lymphocytes exposed to equal concentrations of polymers, alginate or chitosan $(0.156 \mathrm{mg} / \mathrm{ml})$, or CpG ODN $(6 \mu \mathrm{g} / \mathrm{ml})$ are also shown in Figs. 3-5. The percentage of CD4+ T-lymphocytes that naturally express CD69 is very low (3\%). The addition of alginate to cell cultures result in a four-fold increase (13\%) of CD69+ T-lymphocytes (Fig. 3). A similar result (18\%) was obtained with CpG ODN 1826, which correlates well with the above results of size and granularity. In contrast, CD4+ T-lymphocytes responded with elevated CD69 expression levels to the presence of chitosan $(90 \%)$, similar to those observed with the positive control (Con A). The same robust effect of chitosan was also observed in CD8+ T lymphocytes (85\%) (Fig. 4), contrasting with alginate, which has been shown not to induce CD69 expression on CD8+ cells. Therefore, these results are again, more closely related to the granularity and size results shown above. Following CpG ODN 1826 stimulation, we observed about 50\% of CD8+ T-lymphocytes that upregulated CD69; however there was considerable variation in expression (Fig. 4).

The effect of $\mathrm{CpG}$ ODN or the polymers chitosan or alginate on B lymphocyte CD69 activation was also studied. The

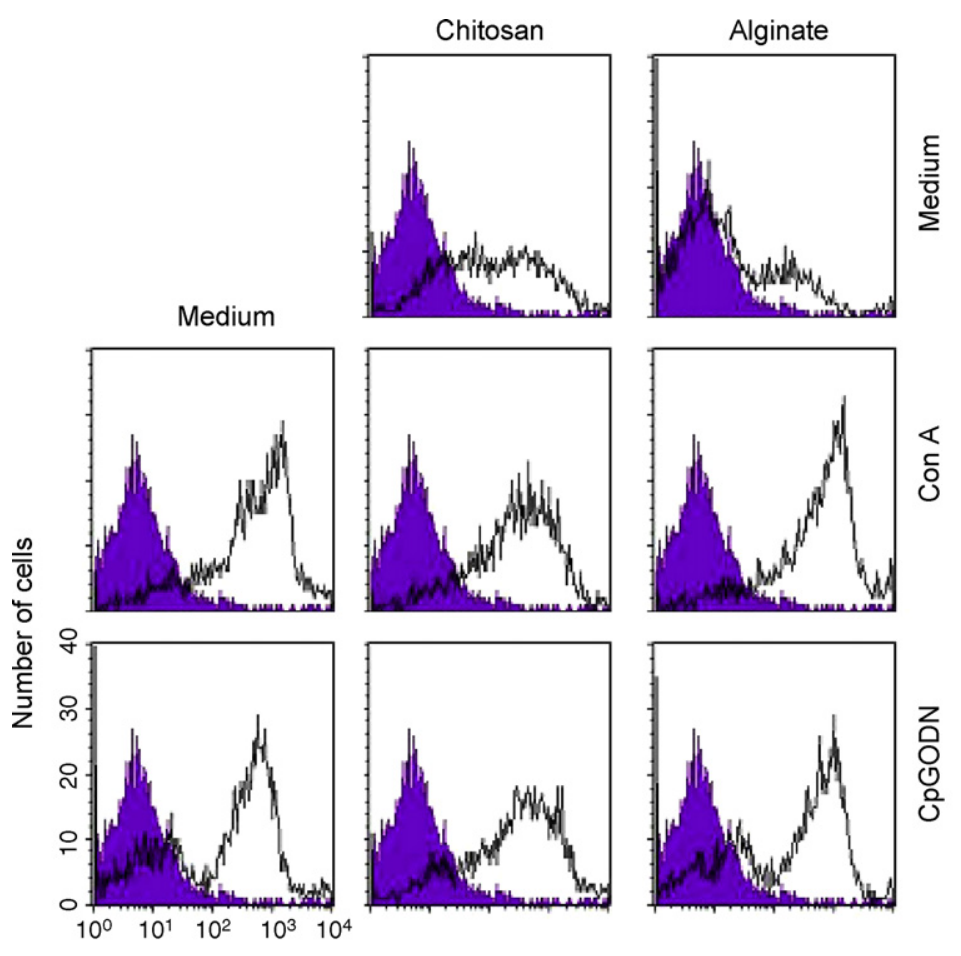

Fluorescence intensity

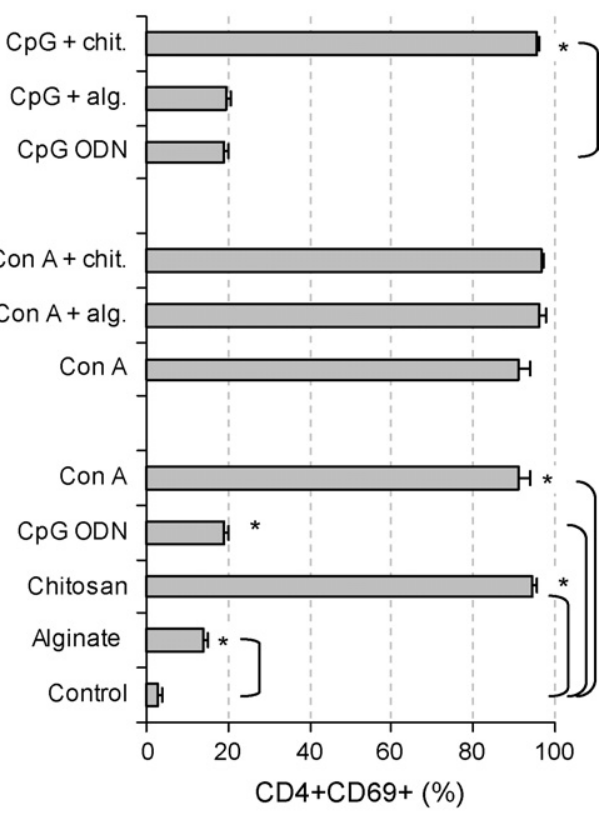

Fig. 3. Effect of the different compounds on the expression of the CD69 receptor on CD4+ splenocytes. The compounds used were the immunostimulators, CpG ODN and Con A and the biopolymers chitosan and alginate. A combination between the biopolymers and the immunostimulators were also used. The filled histograms represent the group control (untreated) and the open histograms the stimulated (pretreated) group. The results are representative of two separate experiments using three animals per experiment and each treatment. The right picture corresponds to the entire data of one experiment. Similar histograms were obtained with the second experiment. The values are mean \pm S.E.M. of the percentage of the positive CD4 cells that express the CD69 antigen, obtained from three different mice. ${ }^{*} p<0.01$. 


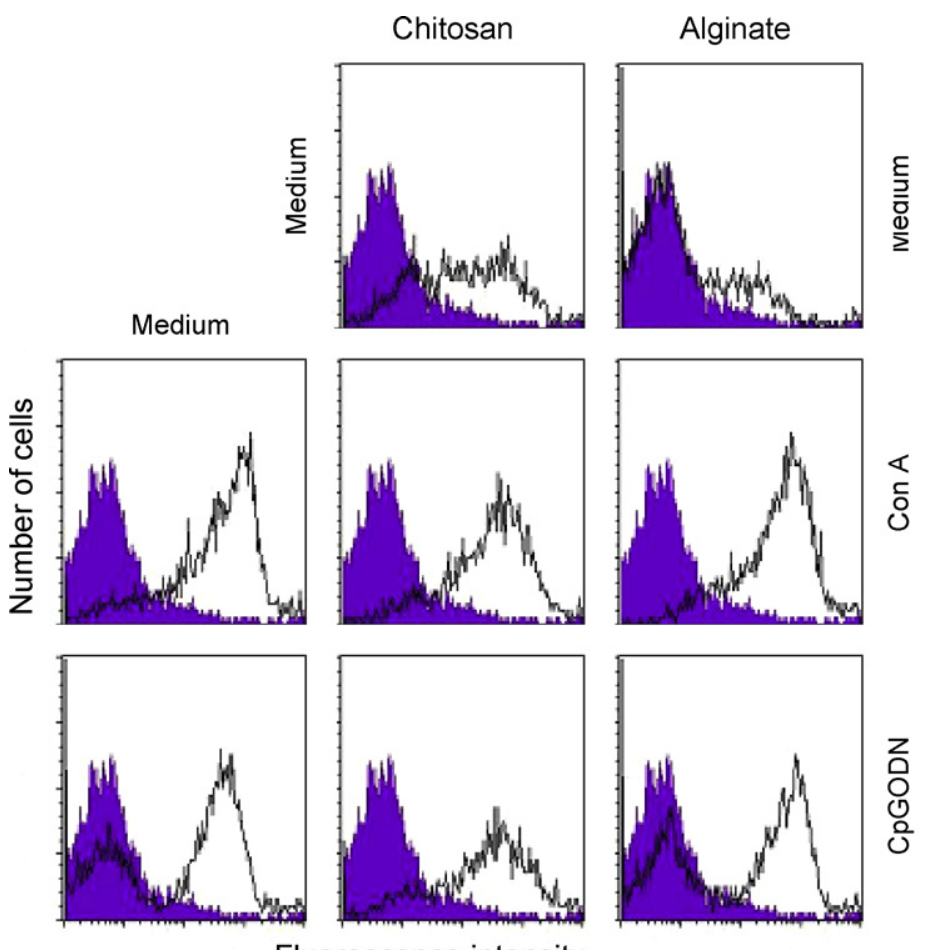

Fluorescence intensity

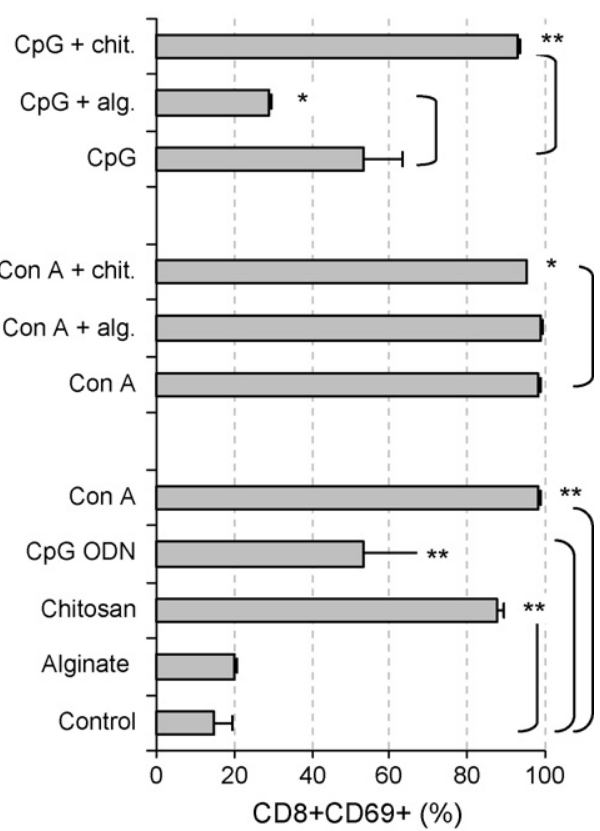

Fig. 4. Effect of the different compounds on the expression of the CD69 receptor on CD8+ splenocytes. The compounds experienced were the immunostimulators, CpG ODN and Con A and the biopolymers chitosan and alginate. A combination of the biopolymers and the immunostimulators were also used. The filled histograms represent the group control (untreated) and the open histograms the stimulated (pretreated) group. The results are representative of two separate experiments using three animals per experiment and each treatment. The right picture corresponds to the entire data of one experiment and similar histograms were obtained with the second experiment. The values are mean \pm S.E.M. of the percentage of the positive CD8 cells that express the CD69 antigen, obtained from three different mice. ${ }^{*} p<0.05 ;{ }^{* *} p<0.01$.

results presented in Fig. 5 show a strong effect of all the assayed biopolymers, with alginate showing the smallest effect (about $40 \%$ ) and chitosan the strongest effect (85\%). More than $95 \%$ of the B cells expressed the CD69 antigen when cultured with CpG ODN, which is in close agreement with a recent published study (Wang and Krieg, 2003). This study was done with two sets of ODN with similar base compositions and it was demonstrated that ODN 1826 is a strong stimulator driving B cells to express CD69 in a dose-dependent fashion. Considering that our results were obtained with lower concentrations of the ODN's and shorter culture times, these results may reflect saturation of the CD69 expression on B cells.

To examine possible synergistic effects using a combination of the polymers chitosan or alginate and the immunostimulator, CpG ODN 1826, the cells were incubated in the presence of both, $\mathrm{CpG}$ and polymers (chitosan or alginate). Chitosan, surprisingly, showed to be a strong CD69 expression inducer for both B and T-lymphocytes, such that CD69 expression could not further be upregulated by $\mathrm{CpG}$. However, a statistically significant difference was observed for the CD8+ T-lymphocyte subpopulation incubated only with chitosan or only with the positive control, Con A. With respect to alginate (Figs. 3 and 4), the results clearly showed that a synergy with $\mathrm{CpG}$ does not occur in T-lymphocyte populations. Furthermore, the presence of alginate in the lymphocyte culture together with $\mathrm{CpG}$ dowregulated the expression of CD69 antigen on CD8+ lymphocytes (Fig. 4) when compared to the cells incubated only with $\mathrm{CpG}$. Potentially, as a result of the saturation of B-lymphocyte CD69 expression induced by $\mathrm{CpG}$, the additional presence of alginate in cell culture did not produce any statistically significant results (Fig. 5).

\subsection{Study of the effect of chitosan and alginate polymers on lymphocyte proliferation}

To study whether the polymers, chitosan or alginate, or the CpG ODN have a proliferative effect on lymphocytes and whether $\mathrm{CpG}$ activates the lymphocytes in synergy with the polymers, mouse spleen lymphocytes were cultured in the presence of the polymers during $96 \mathrm{~h}$. Cells from the spleen are normally resting cells and, when cultured in the absence of a mitogen, do not proliferate (Fig. 6C). In the presence of a mitogen, Con A, the activated cells exhibited the highest values of $\left[{ }^{3} \mathrm{H}\right]$ thymidine uptake. These values are used as the intended positive control (Fig. 6A). On the other hand, as shown in Fig. 6, while $\mathrm{CpG}$ ODN 1826 alone at concentrations of at least $6 \mu \mathrm{g} / \mathrm{ml}$ stimulated splenocytes to proliferate, chitosan and alginate showed no significant stimulation in both concentrations used. However, with higher concentrations of alginate, a large variability of the results was observed (Fig. 6C).

The concomitant presence of the polymers chitosan or alginate and the $\mathrm{CpG}$ or the Con $\mathrm{A}$ in the cell culture did not lead to an increase of $\left[{ }^{3} \mathrm{H}\right]$ thymidine uptake by the cells (Fig. 6A and 

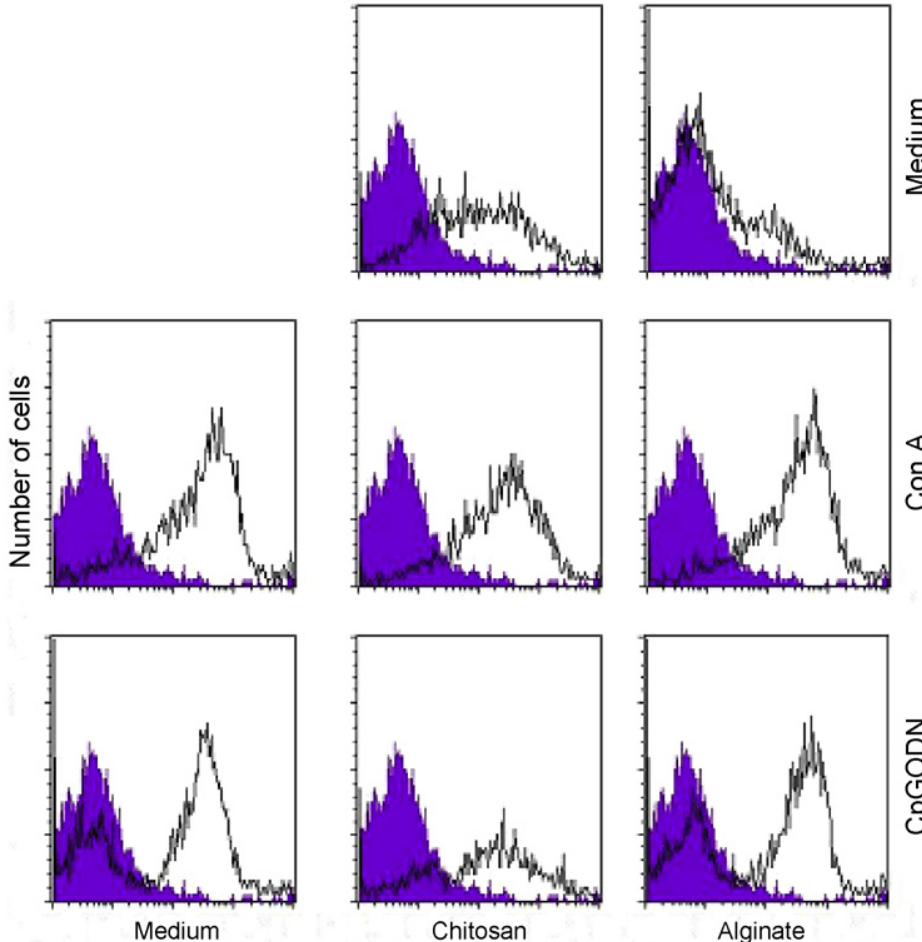

Chitosan
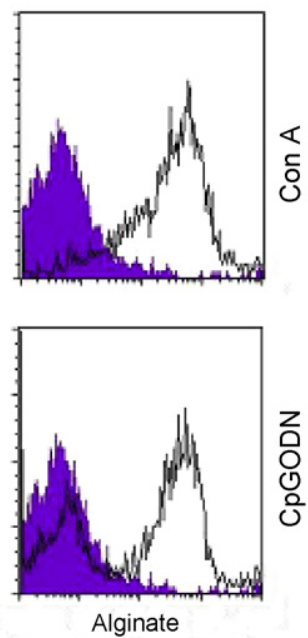

Fluorescence intensity

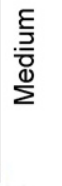

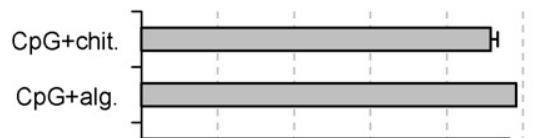
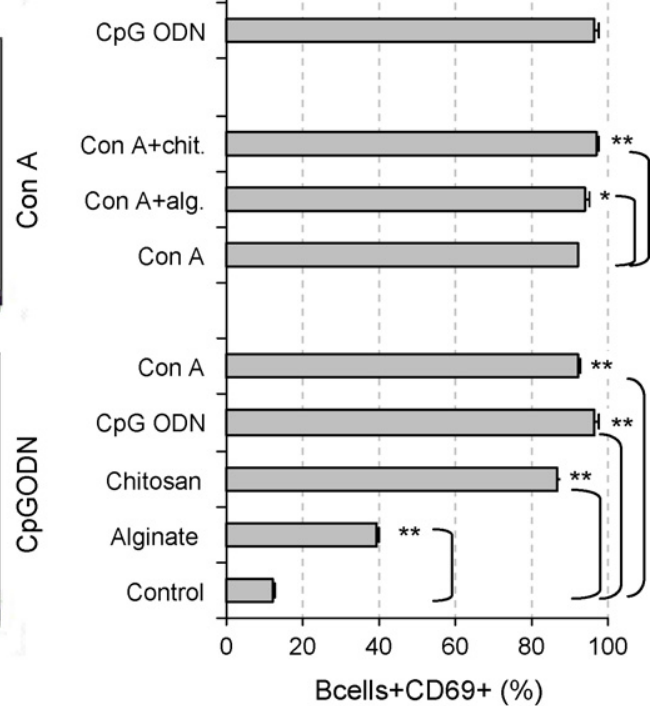

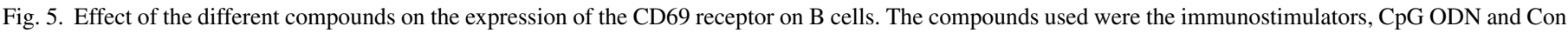

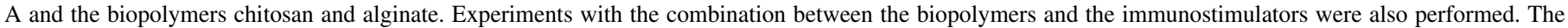

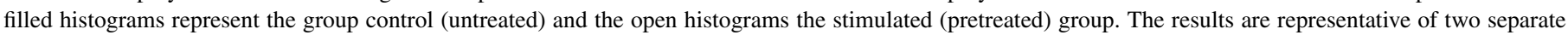

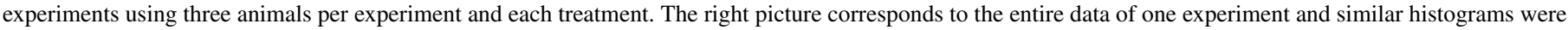

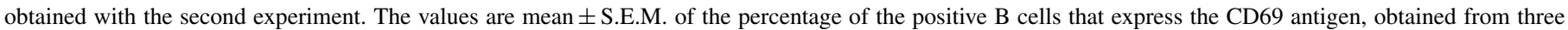
different mice. ${ }^{*} p<0.05 ;{ }^{* *} p<0.01$.

B). These results may possibly indicate that no synergy between $\mathrm{CpG}$ or Con A and each of polymers occurred.

\section{Discussion}

Stimulation of lymphocytes leads to upregulation of various cell surface markers at various stages of cellular activation: CD69 (very early), CD71 (early), CD25 (late), and HLA-Dr (very late) (Reddy et al., 2004). Lymphocytes have little basal expression of CD69, but following activation, CD69 is rapidly expressed ( $2-3 \mathrm{~h})$ in all bone marrow-derived cells except erythrocytes, as reviewed in (Testi et al., 1994; Sancho et al., 2005). The precise role of CD69 in immunity has not been elucidated owing to the absence of a known ligand and adequate in vivo models to study its physiological function (Sancho et al., 2005). It was recently suggested that a transient activation-induced CD69 surface expression may be important for regulating T cell trafficking (Feng et al., 2002). Moreover, CD69 might affect the immune response during T-cell differentiation, involving immunoregulatory cytokines that include, but might not be limited to, TGF- $\beta$, which controls T-cell differentiation. Recent in vivo results indicate that this receptor acts as a regulatory molecule, modulating the inflammatory response (Sancho et al., 2005). Based on several studies, it was reported (Morgan et al., 1999) that CD69 functions as a signal-generating receptor, possibly regulating the activity of the transcription factor AP- 1 and
IL-2 gene expression through both AP-1 and NF-AT complexes. The results presented by Morgan et al. (1999) also suggested that CD69 participates in a variety of processes within the overall activation cascade.

Therefore, in vitro manipulation of early activation marker CD69 is an approach used in many laboratories to explore potential pathways of cellular activation and can be used to measure the immunomodulatory effects of pharmaceutical agents and vaccine antigens (Reddy et al., 2004).

Synthetic oligodeoxynucleotides (CpG ODNs) and bacterial DNA containing unmethylated $\mathrm{CpG}$ dinucleotides in the context of particular base sequences (CpG motifs) are known to mediate several immune responses. In the present study it was shown that CpG ODN 1826 is a potent in vitro CD69 stimulator. Its presence in a culture of mice splenocytes was shown to increase the size and granularity of B lymphocytes, which was accompanied by a strong expression of the CD69 receptor. Furthermore, a mild but statistically significant increase of the expression of CD69 in T-lymphocytes was also observed and was accompanied by high lymphocyte proliferation. These conclusions, however, cannot be generalized to include other CpG ODN, even if the concept of the immunostimulatory $\mathrm{CpG}$ motif has become widely accepted. Recently, it was demonstrated that not all DNA/ODN containing CpG motifs were equally stimulatory (Wang and Krieg, 2003) and CpG 1982 showed itself to be weaker than CpG 1826 in promoting cell activation. CpG ODN 1826 at concentrations of 


\section{(A)}

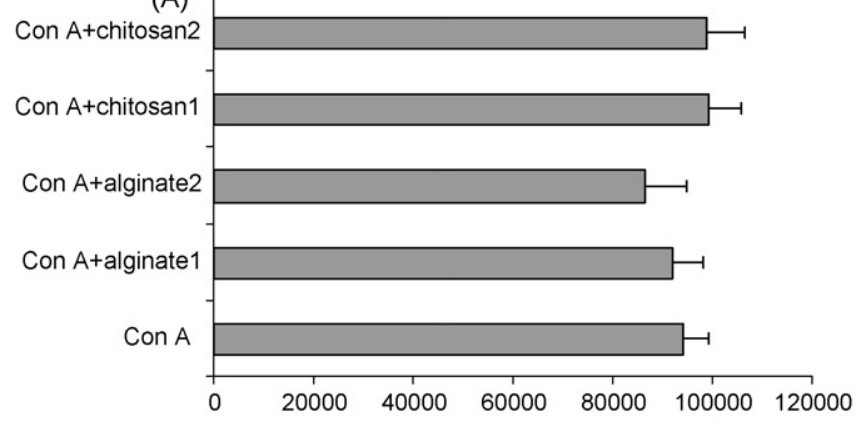

(B) 7
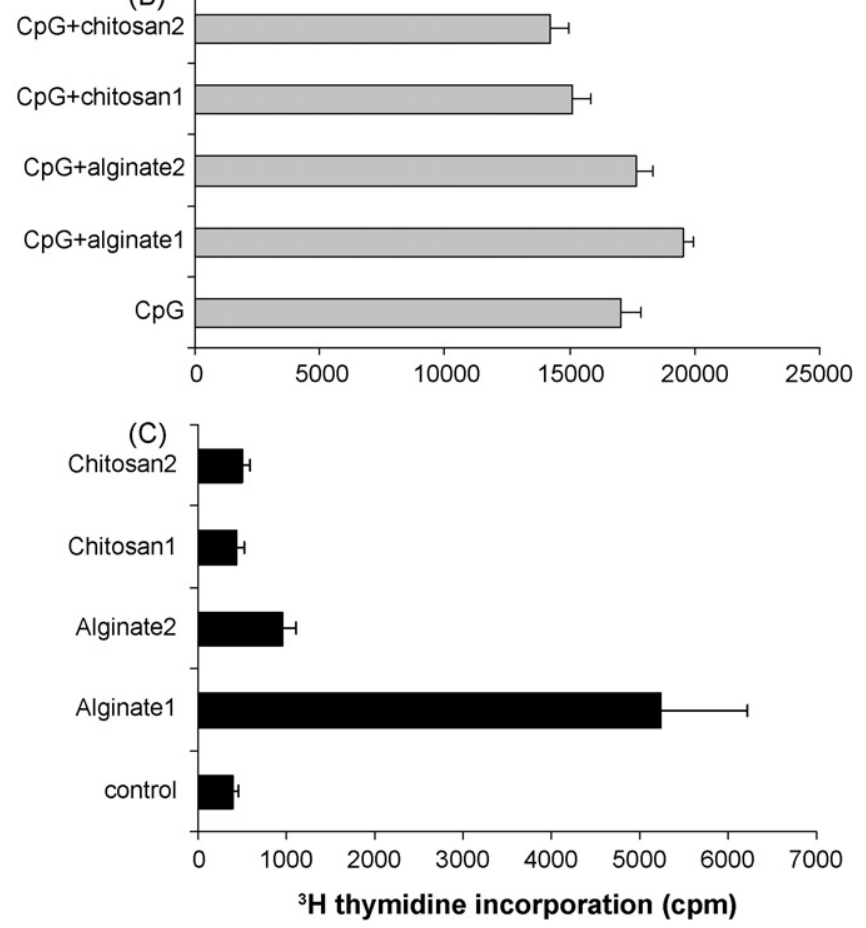

Fig. 6. $\left[{ }^{3} \mathrm{H}\right]$ Thymidine incorporation, expressed in cpm (counts per minute), by the proliferating splenocytes cultured with different compounds for $96 \mathrm{~h}$. The compounds experienced were the immunostimulators, $\mathrm{CpG} \mathrm{ODN} \mathrm{and} \mathrm{Con} \mathrm{A} \mathrm{and}$ two concentrations of the polymers chitosan and alginate. Conc $1=0.312 \mathrm{mg} / \mathrm{ml}$ and conc $2=0.0125 \mathrm{mg} / \mathrm{ml}$. Experiments with the combinations between the biopolymers and the immunostimulators were also performed. Each bar is the mean \pm S.E.M. from three spleen mice cultured in triplicate $(n=9)$. Similar histograms were obtained with the second experiment.

at least $0.3 \mu \mathrm{g} / \mathrm{ml}$ stimulated Ig-Tg B cells to proliferate and drove the same cells to express CD69 in a dose dependent fashion (Wang and Krieg, 2003). Related to T-cell CD69 activation caused by $\mathrm{CpG}$, the data are relatively scarce and in a recent publication (Kranzer et al., 2000), using human T cells, the addition of CpG ODN 2006 to peripheral blood mononuclear cells (PBMC) had only a small variable effect on the CD69 expression by CD4+ or CD8+ T cells. In contrast, the same CpG ODN sequence specifically caused increased expression of CD69 on CD4+ and CD8+ T cells when PBMC were stimulated via $\alpha$ CD3 (Kranzer et al., 2000). The study of possible synergistic effects between the $\mathrm{CpG}$ and other compounds, which could possibly explain some unexpected in vivo results, like broadening of the spectrum of $\mathrm{CpG}$ that primarily stimulate B cells with potential implications for the initiation and regulation of normal and pathologic immune responses, is an important aspect for a better understanding of the mechanisms involved (Wang and Krieg, 2003). Following this idea, the present study failed to show any synergistic effects between $\mathrm{CpG}$ and the polymers chitosan and alginate, respectively, in stimulating lymphocytes to proliferate or to express CD69 antigen. On the other hand, an unexpected result, to our knowledge described for the first time, was obtained with the polymers alone. The polymers proved to be potent CD69 activators, not only for B cells but in case of chitosan, also for T-cells.

In fact, concluding from comparable studies described in the literature with other compounds (Morgan et al., 1999), it is not known whether the expression was stress-induced or rather activation-induced because the observed CD69 expression was not followed by lymphocyte proliferation. On the other hand, it is not known whether CD69 activation is always linked with T-lymphocyte proliferation and incomplete activation might be indicative of anergy (Sancho et al., 2005). Recently we have reported (Borges et al., 2006) an increase in cell permeability for propidium iodide, observed in lymphocytes treated with polymers chitosan and alginate, and in the present study the same effect was confirmed (data not shown). Moreover, in an in vitro study, chitosan was indicated as the cause of a slightly reversible plasma membrane perturbation in Caco-2 cell monolayers (Dodane et al., 1999). This feature is most likely an indication of cellular stress. Nonetheless, chitosan and alginate, in the concentrations studied, did not show a suppressive effect on the lymphocyte proliferation, induced by the mitogens, Con A or CpG nor had any cytotoxic effects on the cells. These observations are important and point out that the presence of these polymers, for instance in an antigen nanocarrier delivery system, might not be able to induce a suppressive lymphocyte proliferative effect, when internalized by a lymphoid organ. On the contrary, and giving as example a recent study (Seferian and Martinez, 2000), where it was shown that chitosan based formulation administered intraperitoneally, together with a weak antigen, elicited antibody responses that were 100 times higher than those observed for the antigen in the absence of the adjuvant.

The second biopolymer in the present work investigated was a low molecular weight alginate, appropriate for pharmaceutical applications, like tissue engineering and vaccine delivery. Unexpectedly, lymphocytes upregulated CD69 expression when the immune cells were maintained in contact with the polymer. Similar to chitosan, our results showed for the first time that alginate can upregulate CD69 expression on B cells and to a lesser extent on CD4+ T cells and had no effect on CD8+ T lymphocytes. Moreover, alginate did not stimulate lymphocytes to proliferate, which is in accordance with other studies found in literature, and the highest individual variability observed has been attributed to their possible content in polyphenolic-like compounds (Orive et al., 2005).

In the present study, chitosan and alginate were shown to activate lymphocytes and induce expression of the CD69, being the chitosan the one that showed the strongest effect. This feature together with the immune properties mentioned above in Section 1 , led us to hypothesize that the adjuvant properties, suggested 
several times for both polymers, could not only be related to their capacity for transport and delivery of the antigen to the lymphoid tissues, but perhaps may be related to their capacity to stimulate immune cells to initiate or simply amplify an immune response specific to the antigen.

\section{Conclusions}

In this study lymphocytes underwent strong activation and rapid CD69 upregulation upon contact with the biopolymers chitosan or alginate. More controlled studies are needed to understand this activation mechanism and the possible consequences for the initiation of an immune response. Moreover, as these are in vitro studies employing mice spleen cells it remains unknown whether the same effects would be observed with human cells or other peripheral lymphoid organs, such as Peyer's patches, following in vivo oral administration.

\section{References}

Babensee, J.E., Paranjpe, A., 2005. Differential levels of dendritic cell maturation on different biomaterials used in combination products. J. Biomed. Mater. Res. A 74, 503-510.

Baudner, B.C., Verhoef, J.C., Giuliani, M.M., Peppoloni, S., Rappuoli, R., Del Giudice, G., Junginger, H.E., 2005. Protective immune responses to meningococcal $\mathrm{C}$ conjugate vaccine after intranasal immunization of mice with the LTK63 mutant plus chitosan or trimethyl chitosan chloride as novel delivery platform. J. Drug Target 13, 489-498.

Bianco, I.D., Balsinde, J., Beltramo, D.M., Castagna, L.F., Landa, C.A., Dennis, E.A., 2000. Chitosan-induced phospholipase A2 activation and arachidonic acid mobilization in P388D1 macrophages. FEBS Lett. 466, 292-294.

Borges, O., Borchard, G., Verhoef, J.C., de Sousa, A., Junginger, H.E., 2005. Preparation of coated nanoparticles for a new mucosal vaccine delivery system. Int. J. Pharm. 299, 155-166.

Borges, O., Cordeiro-da-Silva, A., Romeijn, S.G., Amidi, M., de Sousa, A., Borchard, G., Junginger, H.E., 2006. Uptake studies in rat Peyer's patches, cytotoxicity and release studies of alginate coated chitosan nanoparticles for mucosal vaccination. J. Control Release 114, 348-358.

Davis, H.L., Weeratna, R., Waldschmidt, T.J., Tygrett, L., Schorr, J., Krieg, A.M., 1998. CpG DNA is a potent enhancer of specific immunity in mice immunized with recombinant hepatitis B surface antigen. J. Immunol. 160, 870-876.

Dodane, V., Amin Khan, M., Merwin, J.R., 1999. Effect of chitosan on epithelia permeability and structure. Int. J. Pharm. 182, 21-32.

Felt, O., Buri, P., Gurny, R., 1998. Chitosan: a unique polysaccharide for drug delivery. Drug Dev. Ind. Pharm. 24, 979-993.

Feng, C., Woodside, K.J., Vance, B.A., El-Khoury, D., Canelles, M., Lee, J., Gress, R., Fowlkes, B.J., Shores, E.W., Love, P.E., 2002. A potential role for CD69 in thymocyte emigration. Int. Immunol. 14, 535-544.

Flo, T.H., Ryan, L., Latz, E., Takeuchi, O., Monks, B.G., Lien, E., Halaas, O., Akira, S., Skjak-Braek, G., Golenbock, D.T., Espevik, T., 2002. Involvement of toll-like receptor (TLR) 2 and TLR4 in cell activation by mannuronic acid polymers. J. Biol. Chem. 277, 35489-35495.

George, M., Abraham, T.E., 2006. Polyionic hydrocolloids for the intestinal delivery of protein drugs: alginate and chitosan - a review. J. Control Release $114,1-14$

Jork, A., Thurmer, F., Cramer, H., Zimmermann, G., Gessner, P., Hamel, K., Hofmann, G., Kuttler, B., Hahn, H.J., Josimovic-Alasevic, O., Fritsch, K.G., Zimmermann, U., 2000. Biocompatible alginate from freshly collected $\mathrm{Lam}$ inaria pallida for implantation. Appl. Microbiol. Biotechnol. 53, 224-229.

Kim, B., Bowersock, T., Griebel, P., Kidane, A., Babiuk, L.A., Sanchez, M., Attah-Poku, S., Kaushik, R.S., Mutwiri, G.K., 2002. Mucosal immune responses following oral immunization with rotavirus antigens encapsulated in alginate microspheres. J. Control Release 85, 191-202.
Klinman, D.M., 2004. Immunotherapeutic uses of $\mathrm{CpG}$ oligodeoxynucleotides. Nat. Rev. Immunol. 4, 249-258.

Klock, G., Pfeffermann, A., Ryser, C., Grohn, P., Kuttler, B., Hahn, H.J., Zimmermann, U., 1997. Biocompatibility of mannuronic acid-rich alginates. Biomaterials 18, 707-713.

Kranzer, K., Bauer, M., Lipford, G.B., Heeg, K., Wagner, H., Lang, R., 2000. CpG-oligodeoxynucleotides enhance T-cell receptor-triggered interferon-gamma production and up-regulation of CD69 via induction of antigen-presenting cell-derived interferon type I and interleukin-12. Immunology 99, 170-178.

Maeda, Y., Kimura, Y., 2004. Antitumor effects of various low-molecular-weight chitosans are due to increased natural killer activity of intestinal intraepithelial lymphocytes in sarcoma 180-bearing mice. J. Nutr. 134, 945-950.

Matsunaga, T., Yanagiguchi, K., Yamada, S., Ohara, N., Ikeda, T., Hayashi, Y., 2006. Chitosan monomer promotes tissue regeneration on dental pulp wounds. J. Biomed. Mater. Res. A 76, 711-720.

Mebius, R.E., Kraal, G., 2005. Structure and function of the spleen. Nat. Rev. Immunol. 5, 606-616.

Morgan, C.D., Greene Jr., J.F., Measel Jr., J.W., 1999. Induction of surface antigen CD69 expression in T-lymphocytes following exposure to actinomycin D. Int. J. Immunopharmacol. 21, 689-703.

Mori, T., Murakami, M., Okumura, M., Kadosawa, T., Uede, T., Fujinaga, T., 2005. Mechanism of macrophage activation by chitin derivatives. J. Vet. Med. Sci. 67, 51-56.

Nishimura, K., Nishimura, S., Seo, H., Nishi, N., Tokura, S., Azuma, I., 1987. Effect of multiporous microspheres derived from chitin and partially deacetylated chitin on the activation of mouse peritoneal macrophages. Vaccine 5, 136-140.

O'Hagan, D., 2006. Microparticles as vaccine delivery systems. In: Schijins, V., O'Hagan, D. (Eds.), Immunopotentiators in Modern Vaccines, vol. 1. Academic Press, pp. 123-147.

Orive, G., Carcaboso, A.M., Hernandez, R.M., Gascon, A.R., Pedraz, J.L., 2005. Biocompatibility evaluation of different alginates and alginate-based microcapsules. Biomacromolecules 6, 927-931.

Otterlei, M., Sundan, A., Skjak-Braek, G., Ryan, L., Smidsrod, O., Espevik, T., 1993. Similar mechanisms of action of defined polysaccharides and lipopolysaccharides: characterization of binding and tumor necrosis factor alpha induction. Infect. Immun. 61, 1917-1925.

Porporatto, C., Bianco, I.D., Correa, S.G., 2005. Local and systemic activity of the polysaccharide chitosan at lymphoid tissues after oral administration. J. Leukoc. Biol. 78, 62-69.

Read, R.C., Naylor, S.C., Potter, C.W., Bond, J., Jabbal-Gill, I., Fisher, A., Illum, L., Jennings, R., 2005. Effective nasal influenza vaccine delivery using chitosan. Vaccine 23, 4367-4374.

Reddy, M., Eirikis, E., Davis, C., Davis, H.M., Prabhakar, U., 2004. Comparative analysis of lymphocyte activation marker expression and cytokine secretion profile in stimulated human peripheral blood mononuclear cell cultures: an in vitro model to monitor cellular immune function. J. Immunol. Methods 293, 127-142.

Sancho, D., Gomez, M., Sanchez-Madrid, F., 2005. CD69 is an immunoregulatory molecule induced following activation. Trends Immunol. 26, 136-140.

Seferian, P.G., Martinez, M.L., 2000. Immune stimulating activity of two new chitosan containing adjuvant formulations. Vaccine 19, 661-668.

Shibata, Y., Foster, L.A., Metzger, W.J., Myrvik, Q.N., 1997. Alveolar macrophage priming by intravenous administration of chitin particles, polymers of $N$-acetyl-D-glucosamine, in mice. Infect. Immun. 65, 1734-1741.

Suckow, M.A., Jarvinen, L.Z., HogenEsch, H., Park, K., Bowersock, T.L., 2002. Immunization of rabbits against a bacterial pathogen with an alginate microparticle vaccine. J. Control Release 85, 227-235.

Tafaghodi, M., Sajadi Tabassi, S.A., Jaafari, M.R., 2006. Induction of systemic and mucosal immune responses by intranasal administration of alginate microspheres encapsulated with tetanus toxoid and CpG-ODN. Int. J. Pharm. $319,37-43$.

Testi, R., D’Ambrosio, D., De Maria, R., Santoni, A., 1994. The CD69 receptor: a multipurpose cell-surface trigger for hematopoietic cells. Immunol. Today $15,479-483$.

Ueno, H., Mori, T., Fujinaga, T., 2001. Topical formulations and wound healing applications of chitosan. Adv. Drug Deliv. Rev. 52, 105-115. 
van der Lubben, I.M., Kersten, G., Fretz, M.M., Beuvery, C., Coos Verhoef, J., Junginger, H.E., 2003. Chitosan microparticles for mucosal vaccination against diphtheria: oral and nasal efficacy studies in mice. Vaccine 21, $1400-1408$.

van der Lubben, I.M., Verhoef, J.C., Borchard, G., Junginger, H.E., 2001. Chitosan for mucosal vaccination. Adv. Drug Deliv. Rev. 52, 139-144.
Wang, Y., Krieg, A.M., 2003. Synergy between CpG- or non-CpG DNA and specific antigen for B cell activation. Int. Immunol. 15, 223-231.

Xu, W., Shen, Y., Jiang, Z., Wang, Y., Chu, Y., Xiong, S., 2004. Intranasal delivery of chitosan-DNA vaccine generates mucosal SIgA and anti-CVB3 protection. Vaccine 22, 3603-3612. 